\title{
The Influence of Medical Jargon Mixed with Foreign Terminology in the Japanese Clinical Environment
}

\author{
Yasuharu Tokuda ${ }^{1}$, Sawako Okamoto ${ }^{2}$, Yasuo Yoshioka ${ }^{3}$, Masao Aizawa $^{3}$, Makiro Tanaka $^{3}$, \\ Kazuhisa Motomura ${ }^{4}$ and Keiko Hayano ${ }^{5}$
}

\begin{abstract}
Objectives To identify patient reports about their difficulties with medical jargon, to classify the most problematic types, and to examine the socio-demographic factors associated with them.

Methods A cross-sectional nationwide survey (October 6 and November 4, 2004) was conducted. Out of 4,500 Japanese people (aged 15 years and older) who had seen physicians, 3,090 agreed to participate (response rate: 69\%). Participants were asked the following: "Do you find physicians that use medical jargon difficult to understand?" and "What type of words did your physician use that required further explanation or clarification?"

Results Of 3,090 respondents, 1,117 participants (36.1\%; 95\% confidence interval, 34.5-37.8\%) reported difficulties understanding medical jargon. Those between the ages of 30 and 49 years, self-employed workers, homemakers, and unemployed individuals experienced the most difficulties. Difficult jargon included: 1) technical Japanese words, such as Kakutan Saibo-shin (sputum cytology) (57\% of participants); 2) English medical terminology, such as clinical path (57\%); and 3) English medical abbreviations, such as EBM (47\%). Conclusions In addition to avoiding technical words when communicating with their patients, Japanese physicians should consider the unique medical situation in which foreign terminology and abbreviations are used in Japan. Translation of foreign terminology into Japanese can be helpful for patients. Physicians should take the initiative to educate patients and familiarize them with foreign terminology and abbreviations.
\end{abstract}

Key words: medical terminology, medical abbreviation, foreign terminology, clinical communication, medical interview

(Inter Med 47: 1329-1334, 2008)

(DOI: 10.2169/internalmedicine.47.0862)

\section{Introduction}

Various skills and techniques can be used to promote effective clinical communication between patients and physicians, such as structuring communication, addressing a patient's agenda, being empathetic, using non-verbal skills, and considering cultural tendencies. Medical jargon constitutes an additional obstacle for patient understanding, especially when the following factors are considered:

- Immediately after a clinical encounter, patients generally remember only about $60 \%$ of the information provided by their physician and this value drops to about $50 \%$ several weeks later (1).

- When patients are asked to explain their illness and treatment, they are generally able to correctly identify only about $50 \%$ of the critical information (2).

- Only $50 \%$ of patients can adequately explain their medications (2). Even though patients have problems understanding physician's explanations containing medical jargon, physicians still tend to expect their patients to adhere to the treatment plan (3). Physicians may be under the assumption that patients are as familiar with medical jargon as they are (4).

\footnotetext{
${ }^{1}$ Department of Medicine, St. Luke's International Hospital, Tokyo, ${ }^{2}$ Tokyo Medical and Dental University, Tokyo, ${ }^{3}$ The National Institute for Japanese Language, Tachikawa, ${ }^{4}$ Ohji Seikyo Hospital, Tokyo and ${ }^{5}$ Department of General Medicine, Kumamoto University School of Medicine, Kumamoto

Received for publication December 28, 2007; Accepted for publication April 30, 2008

Correspondence to Dr. Yasuharu Tokuda, tokuyasu@orange.ocn.ne.jp
} 
Because of these factors, medical jargon tends to slip easily into clinical communications (1). Moreover, communication difficulties appear to be closely associated with patient dissatisfaction, threats to patient safety, and a higher incidence of malpractice claims (5-10). To identify the most difficult types of medical jargon, we examined patient-reported experiences with medical jargon as well as sociodemographic factors associated with these difficulties.

\section{Methods}

\section{Study population}

Authors (Yoshioka, Aizawa, and Tanaka) in this study originally developed and used the questionnaire in an educational project designed to improve linguistic communication among Japanese laypersons. That study was supported by a grant from the Ministry of Education, Culture, Sports, Science and Technology (Grant \#17520310). The same questionnaire was used in the present study whose purpose was to identify problems related to verbal communication between patients and physicians in Japan.

The survey population consisted of individuals 15 years of age and older living in Japan. Research subjects were randomly chosen through probability proportionate to size sampling based on household addresses registered in all Japanese municipalities. Specifically, the sampling method involved two stages. First, sampling areas were selected randomly, using the method of probability proportionate to size. The higher population wards were given a higher probability of being selected, ensuring that the sample was geographically representative of the municipalities. Second, within a ward identified for sampling, the households were selected randomly and contacted around the randomly selected starting points. Out of 4,500 people, 3,090 respondents (response rate: $69 \%$ ) voluntarily agreed to participate in this study. No financial incentives were offered. This study was approved by the Ethics Committee of the Kumamoto University School of Medicine in Japan.

\section{Data collection}

With the participants' permission, study interviewers visited households of 3,090 study subjects between October 6 and November 4, 2004. Trained interviewers, who belonged to the Japan New Information Center (Tokyo, Japan), visited each household and began each face-to-face interview session by explaining the purpose and method of the study. Participants were asked questions related to their daily communication in Japan, such as words used by the media, foreign words, and words used during polite exchanges. In addition to questions about these general communication issues, the following questions were included in the survey: "Do you find physicians that use medical jargon difficult to understand?" and "What type of words did your physician use that required further explanation or clarification?" In addition, the participants were asked to choose several words from a pre-selected list of the words in each type, including technical words, foreign medical terminology, and medical abbreviations.

Demographic and socio-economic data were also collected. Demographic data included age, gender, and area of residence. Each community was scored in one of four categories: 1) large city with a population of more than 1 million; 2) city with a population between 100,000 and 1 million; 3) city or town with a population between 5,000 and 100,000 ; and, 4) rural area or village with a population of less than 5,000 .

Socio-economic data included annual household income, educational credentials and occupational status. The annual household income was split into 6 categories: 1) less than 2 million Japanese yen; 2) 2 to 4 million yen; 3) 4 to 6 million yen; 4) 6 to 8 million yen; 5) 8 to 10 million yen; and; 6) 10 million yen and higher. Occupational status was assessed using 10 categories: 1) professionals; 2) managers; 3) clerks; 4) self-employed workers; 5) service-industry workers; 6) farmers; 7) laborers; 8) homemakers; 9) students; and 10) unemployed. Education credentials were classified into 3 levels: 1) junior high school graduate or less; 2) high school graduate; or, 3) college graduate or more.

\section{Statistical Analyses}

A 95\% confidence interval (CI) was applied to examine difficulties with understanding medical jargon regarding demographic and socioeconomic variables. To identify significant factors associated with these difficulties, we used a multivariate logistic regression model adjusted for the following demographic and socio-economic covariates: age, gender, area of residence, annual household income, employment, and education. Statistical significance was less than a two-tailed level value of $0.05(\mathrm{p}<0.05)$. SPSS software version 15.0J (SPSS Japan, Tokyo) was used for all statistical analyses.

\section{Results}

The mean age of participants was 50 years (range: 15-94). Respondents included $46 \%$ males and $54 \%$ females. Of the total participants, 36.1\% (95\% CI, 34.5-37.8\%) experienced difficulties understanding medical jargon in clinical communications with physicians. Table 1 shows how the results varied by demographic and socioeconomic characteristics based on a multivariate logistic regression analysis.

By age, those aged 30-39 $(44.3 \%, \mathrm{p}<0.01)$ and aged $40-$ $49(43.3 \%, \mathrm{p}<0.05)$ showed a significantly higher incidence of experiencing difficulties with medical jargon. In terms of community, those who lived in areas of 5,000-100,000 population $(37.6 \%)$ showed the highest level of difficulties, but this result was not statistically significant. By income, those who earned from $6,000,000$ to $8,000,000$ yen $(39.7 \%$ ) had the most concerns about medical jargon, but this result was not statistically significant. In terms of employment status, a statistically significant higher incidence was shown 
Table 1. Patient-reported Difficulties in Understanding Medical Jargon (n=3,090)

\begin{tabular}{|c|c|c|c|c|}
\hline \multirow[t]{2}{*}{ Variable } & \multirow{2}{*}{$\begin{array}{c}\text { Participants } \\
\mathrm{n}(\%)\end{array}$} & \multicolumn{2}{|c|}{ Experienced Difficulty } & \multirow[t]{2}{*}{ p-value } \\
\hline & & $\mathrm{n}$ & $\%(95 \% \mathrm{CI})$ & \\
\hline All participants & 3090 & 1,117 & $36.1(34.5-37.8)$ & \\
\hline Demographics & $\mathrm{n}=3090$ & & & \\
\hline \multicolumn{5}{|l|}{ Gender } \\
\hline Male & $1421(46.0)$ & 501 & $35.3(32.8-37.7)$ & RG* \\
\hline Female & $1669(54.0)$ & 616 & $36.9(34.6-39.2)$ & NS \\
\hline \multicolumn{5}{|l|}{ Age } \\
\hline $15-29$ & $411(13.3)$ & 106 & $25.8(21.5-30.0)$ & $\mathrm{RG}^{*}$ \\
\hline $30-39$ & $508(16.4)$ & 225 & $44.3(40.0-48.6)$ & $<0.01$ \\
\hline $40-49$ & $476(15.4)$ & 206 & $43.3(38.8-47.7)$ & $<0.05$ \\
\hline $50-59$ & $619(20.0)$ & 226 & $36.5(32.7-40.3)$ & NS \\
\hline $60-69$ & $625(20.2)$ & 196 & $31.4(27.7-35.0)$ & NS \\
\hline$>=70$ & $451(14.6)$ & 158 & $35.0(30.6-39.5)$ & NS \\
\hline \multicolumn{5}{|l|}{ Area of Residence } \\
\hline Population $>1,000,000$ & $686(22.2)$ & 256 & $37.3(33.7-40.9)$ & RG* \\
\hline Population of $100,000-1,000,000$ & $1146(37.1)$ & 412 & $36.0(33.2-38.7)$ & NS \\
\hline Population $<100,000$ & $596(19.3)$ & 224 & $37.6(33.7-41.5)$ & NS \\
\hline Rural area & $662(21.4)$ & 225 & $34.0(30.4-37.6)$ & NS \\
\hline \multicolumn{5}{|l|}{ Socioeconomic characteristics } \\
\hline Annual household income (Japanese yen) & $\mathrm{n}=2291$ & & & \\
\hline$<2,000,000$ & $238(7.7)$ & 84 & $35.3(29.2-41.4)$ & RG* \\
\hline $2,000,000$ to $<4,000,000$ & $605(19.6)$ & 225 & $37.2(33.3-41.1)$ & NS \\
\hline $4,000,000$ to $<6,000,000$ & $608(19.7)$ & 228 & $37.5(33.6-41.4)$ & NS \\
\hline $6,000,000$ to $<8,000,000$ & $418(13.5)$ & 166 & $39.7(35.0-44.4)$ & NS \\
\hline $8,000,000$ to $<10,000,000$ & $228(7.4)$ & 87 & $38.2(31.8-44.5)$ & NS \\
\hline$>=10,000,000$ & $194(6.2)$ & 72 & $37.1(30.3-44.0)$ & NS \\
\hline Employment status & $n=3090$ & & & \\
\hline Student & $177(5.7)$ & 34 & $19.2(13.3-25.1)$ & RG* \\
\hline Clerk & $518(16.8)$ & 215 & $41.5(37.2-45.8)$ & NS \\
\hline Service Worker & $307(9.9)$ & 91 & $29.6(24.5-34.8)$ & NS \\
\hline Agriculture, Forestry and Fishery Worker & $131(4.2)$ & 43 & $32.8(24.7-41.0)$ & NS \\
\hline Laborer & $579(18.7)$ & 194 & $33.5(29.6-37.4)$ & NS \\
\hline Professional & $31(1.0)$ & 15 & $48.4(29.8-67.0)$ & NS \\
\hline Manager & $71(2.3)$ & 28 & $39.4(27.8-51.1)$ & NS \\
\hline Homemaker & $776(25.1)$ & 304 & $39.2(35.7-42.6)$ & $<0.05$ \\
\hline Self-employed & $45(1.5)$ & 20 & $44.4(29.3-59.5)$ & $<0.05$ \\
\hline Unemployed & $455(14.7)$ & 173 & $38.0(33.5-42.5)$ & $<0.05$ \\
\hline Education & $n=3049$ & & & \\
\hline Junior High or Less & $585(18.9)$ & 190 & $32.5(28.7-36.3)$ & $\mathrm{RG}^{*}$ \\
\hline High School Graduate & $1555(50.3)$ & 568 & $36.5(34.1-38.9)$ & NS \\
\hline College Degree or More & $909(29.4)$ & 352 & $38.7(35.6-41.9)$ & NS \\
\hline
\end{tabular}

$\mathrm{CI}=$ confidence interval. $\mathrm{NS}=$ not significant.

All variables were analyzed as categorical variables in regression model: *RG indicates the reference group.

for self-employed workers $(44.4 \%, \mathrm{p}<0.05)$, homemakers $(39.2 \%, \mathrm{p}<0.05)$, and the unemployed $(38.0 \%, \mathrm{p}<0.05)$. In terms of education, those who obtained college degrees or higher (38.7\%) showed the highest level of difficulty with medical jargon, but this result was not statistically significant.

Participants identified the following words spoken by physicians as being difficult to understand (Table 2): 1) Japanese technical words such as Kakutan Saibo-shin (sputum cytology) and Himatsu Kansen (droplet infection) (57\% of the participants); 2) foreign medical terminology, such as triage and clinical path (57\%); 3) foreign medical abbreviations, such as $E B M$ (evidence-based medicine) and $A E D$ (automated external defibrillator) (47\%); and, 4) Japanese words commonly used in hospitals such as Shohosen (prescription) and Shinsatsu (physical examination) (27\%). Table 3 presents a listing of the five most difficult medical terms reported by participants.

\section{Discussion}

More than one-third of participants in this study reported experiencing difficulties understanding medical jargon in clinical communications with their physicians. The several types of factors associated with a greater likelihood of reporting the difficulties may be summarized into two major factors: physician-related factors and patient-related factors. Physician-related factors include the use of Japanese technical words, and foreign medical terminology and abbreviations, especially English technical terms. Patient-related factors include demographics and socio-economic influences. Those between 30 and 49 years of age, self-employed workers, homemakers, and the unemployed had statistically significant difficulty understanding medical jargon. 
Table 2. Classification of Medical Jargon that is Difficult for Japanese Patients

\begin{tabular}{|c|c|c|c|c|c|c|}
\hline \multirow[b]{2}{*}{ Technical Words } & \multicolumn{3}{|c|}{ Experienced Difficulty } & \multicolumn{3}{|c|}{$95 \% \mathrm{CI}$} \\
\hline & $57.1 \%$ & ( & $55.3 \%$ & , & $58.8 \%$ & ) \\
\hline Foreign Medical Terminology & $56.5 \%$ & ( & $54.7 \%$ & , & $58.2 \%$ & ) \\
\hline Medical Abbreviations & $47.3 \%$ & ( & $45.5 \%$ & , & $49.0 \%$ & ) \\
\hline Common Words used in Hospitals & $27.3 \%$ & ( & $25.7 \%$ & , & $28.9 \%$ & ) \\
\hline
\end{tabular}

$\mathrm{CI}=$ confidence interval.

Table 3. List of the Top Five Medical Terms that are Difficult for Japanese Patients

\begin{tabular}{cccc}
\hline Rank & Technical Words & Foreign Medical Terminology & Medical Abbreviations \\
\hline 1 & Sputum Cytology & Triage & EBM \\
2 & Droplet Infection & Clinical Path & AED \\
3 & Decubitus & Recipient & ADHD \\
4 & Aspiration & Living Will & PTSD \\
5 & Prognosis & Primary Care & CT \\
\hline
\end{tabular}

$\mathrm{EBM}=$ evidence-based medicine; $\mathrm{AED}=$ automated external defibrillator;

$\mathrm{ADHD}=$ attention-deficit hyperactivity disorder; $\mathrm{PTSD}=$ post-traumatic stress disorder:

$\mathrm{CT}=$ computed tomography.

As for physician-related factors, even Japanese words commonly used in the clinical environment seem to be unfamiliar to patients or their use causes them to feel uninformed and embarrassed. Therefore, it is generally accepted that physicians should avoid medical terminology and explain medical conditions in simple and ordinary language to create better relationships with their patients $(2,11)$.

Medical terminology and abbreviations adapted from foreign languages - especially English - are commonly used in the Japanese clinical environment. Physicians use foreign language terminology and abbreviations because they may be unable to find appropriate and concise translations to convey their proper meaning. Terminology developed in Western nations that is translated into Japanese can be even more complicated and confusing for patients. Thus, physicians may opt to use the original foreign abbreviations and terminology in the Japanese medical environment. However, the aforementioned terminology may increase uncertainties when Japanese patients communicate with physicians. Since physicians and patients do not share the same language familiarity during patient-physician communications, patients may feel awkward using foreign medical terminology and abbreviations $(5,12)$.

As for patient-related socio-demographics factors, participants aged 30-49, self-employed workers, homemakers, and the unemployed had greater difficulty in understanding medical jargon. People aged 30-49 may have higher expectations for communicating with physicians than do the young or elderly. The age group younger than 30 years old and a generally healthier generation may have less experience dealing with physicians, whereas the elderly "may engage in self-fulfilling behavior that reflects the limitations being imposed upon [the elderly-selves]" (13). Those aged 30-49 years have more opportunities for direct medical contacts as they support children and aging parents. In addition, globalization in technology and the economy has accelerated intercultural contact and may have changed the Japanese communication style (14). The generation of 30-49 years old also is more likely to speak up and communicate their needs to physicians. Therefore, they may feel a greater degree of frustration when communicating with physicians who use medical jargon.

In Japan, most self-employed workers run businesses and their physical well-being may be an important factor in their ability to be financially secure and to support their families and employees. Japanese self-employed workers may be conscientious and motivated to learn about their health status and may place more value on patient-physician communication. The use of medical jargon may hinder their understanding.

Females are the majority of homemakers in Japan. Females are generally actively involved in exchanging information by asking questions and showing interest in others' ideas (15). Wood has argued that women desire oral communication to build trust with others, share their feelings and thoughts, and learn new things through disclosure. They also tend to be responsive to others (15). Ankem also stated that females indicate a greater need for information related to their treatment (16). The use of medical jargon can be an obstacle to women who adopt this more communicative style in clinical communications, and may cause them to feel uncomfortable, uninformed, and excluded.

As for the unemployment status, jobless participants could have come from a number of different occupations, demographic categories, education levels, and psychological states. Their condition of unemployment may be temporary or permanent. In relatively homogeneous cultures, such as Japan, people tend to show a high-degree of uncertainty avoidance (12). In addition to the insecurity created by being jobless, it is possible that medical jargon may increase 
uncertainty among these participants. Increasing uncertainty could make the unemployed participants feel uninformed and embarrassed.

This study has several limitations. There may be recall bias because the questionnaires were given in-person by researchers who visited each participant. Also, we did not ask participants to report on the frequency of their difficulties understanding medical jargon when communicating with physicians. To gain an objective picture of how medical jargon interferes with the understanding of Japanese patients, a study using direct observation of patient-physician communications may be valuable. Lastly, we had no information related to the medical condition of the patient participants, such as disease, frequency of visits, and health literacy. Ability to read, listen, and comprehend health information is now considered as a vital element of maintaining and improving health, although health care systems are only beginning to recognize the issue of health literacy and take action to overcome its negative impact (17-19). Further studies are needed to explore possible associations between these factors and difficulties in understanding medical jargon. However, this study provides the insightful quantitative research from an objective viewpoint into the patients' reactions for medical jargon in Japan.

In addition to terms generally used in the Japanese medical setting, foreign medical terminology and abbreviations seem to disrupt Japanese patients' understanding in clinical communications with physicians and thus become obstacles for promoting effective communication. Such miscommunication may also generate frustration in patients, which may become "a barrier to expected goal attainment" (20). Although a goal in medicine is to obtain satisfactory outcomes, difficulties understanding conversations with physicians would negatively affect building a trusting relationship and obtaining a satisfactory medical outcome.

Patients of physicians who have better communication skills are more likely to understand their conditions and adhere to treatment recommendations $(21,22)$, and to be more satisfied with their care $(1,10)$. Thus, it is important to educate and train Japanese physicians to communicate with patients using simple, lay terminology. Although communication programs in the Japanese medical education system have existed since December 2005, more careful attention must be paid to unique medical environments in which Japanese technical and foreign terms and abbreviations are inextricably interwoven. The communication program in the medical education system in Japan should be tailored to fit these unique environments.

Numerous studies have found that patient education and interventions can improve patient understanding, increase patient literacy, and promote patient participation in their own healthcare and decision-making processes $(18,23,24)$. In addition to improved communication skills training programs for physicians, intervention programs for patients can increase their familiarity with, and understanding of foreign terms and abbreviations that are interlaced in the Japanese medical environment and are difficult to translate into Japanese.

\section{Acknowledgement}

This study was supported by a Research Grant from the Ministry of Health, Labor, and Welfare of the Japanese Government. The funding source had no role in data collection and analysis of this study. There are no conflicts of interest to disclose. The authors gratefully acknowledge Dr. Kelly Monastiriakos, Mr. Todd M. Crick, and Ms. Clara Marin for editing.

\section{References}

1. Coulehan JL, Block MR. Negotiation and the Healing Connection. In: The medical interview: mastering skills for clinical practice. 2 nd ed. F. A. Davis Co, Philadelphia, 1992: 301-325.

2. Cohen-Cole SA. Why "Three" Functions? In: The medical interview: the three-function approach. 1st ed. Mosby, St. Louis, 1991: 4-10.

3. Makoul G, Arntson P, Schofield T. Health promotion in primary care: physician-patient communication and decision making about prescription medications. Soc Sci Med 41: 1241-1254, 1995.

4. Aldrich CK. A brief preview of history-taking. In: The medical interview: gateway to the doctor-patient relationship: a manual for first or second year medical student. Partheon Pub. Group, New York, 1993: 95-110.

5. Itoda N, Nagano C, Tsutsui T, Hoorie M. Risk communication using medical terminology in Japanese high school students. Japanese Journal of Occupational Health 47: 861, 2005.

6. Lester GW, Smith SG. Listening and talking to patients. A remedy for malpractice suits? West J Med 158: 268-272, 1993.

7. Levinson W, Roter DL, Mullooly JP, Dull VT, Frankel RM. Physician-patient communication. The relationship with malpractice claims among primary care physicians and surgeons. JAMA 277: 553-559, 1997.

8. Sutcliffe KM, Lewton E, Rosenthal MM. Communication failures: an insidious contributor to medical mishaps. Acad Med 79: 186194, 2004.

9. Keyes C. Quality of provider-patient communication. Int J Qual Health Care 12: 347, 2000.

10. Williams N, Ogden J. The impact of matching the patient's vocabulary: a randomized control trial. Fam Pract 21: 630-635, 2004.

11. Beck RS, Daughtridge R, Sloane PD. Physician-patient communication in the primary care office: a systematic review. J Am Board Fam Pract 15: 25-38, 2002.

12. Gudykunst WB. Anxiety/uncertainty management (AUM) theory: Current status. In: Intercultural Communication Theory. Wiseman R, Ed. Sage Publications Inc, Thousand Oaks, CA, 1995: 8.

13. Samovar L, Porter R. Intercultural Communication: A reader. In: Understanding the co-culture of the elderly. McKay V, Ed. Wadsworth Publishing, Belmont, CA, 2000: 180-89.

14. Samovar L, Porter R, McDaniel E. Understanding intercultural communication. In: Intercultural Communication: A Reader. Samovar L, Porter R, Eds. Wadsworth Publishing, Belmont, CA, 2006.

15. Wood J. Gender, communication, and culture. In: Intercultural Communication: A Reader, 8th ed. Samovar L, Porter R, Eds. Wadsworth, Belmont, CA, 1997: 170-179. 
16. Ankem K. Information-seeking behavior of women in their path to an innovative alternate treatment for symptomatic uterine fibroids. J Med Libr Assoc 95: 164-172, 2007.

17. Gazmararian JA, Williams MV, Peel J, Baker DW. Health literacy and knowledge of chronic disease. Patient Educ Couns 51: 267275, 2003.

18. Safeer RS, Cooke CE, Keenan J. The impact of health literacy on cardiovascular disease. Vasc Health Risk Manag 2: 457-464, 2006.

19. Wolf MS, Gazmararian JA, Baker DW. Health literacy and health risk behaviors among older adults. Am J Prev Med 32: 19-24, 2007.

20. Berkowitz L. Aggression: its causes, consequences, and control. McGraw-Hill, New York, 1993.
21. Cegala DJ. A study of doctors' and patients' communication during a primary care consultation: implications for communication training. J Health Commun 2: 169-194, 1997.

22. Joos SK, Hickam DH, Gordon GH, Baker LH. Effects of a physician communication intervention on patient care outcomes. J Gen Intern Med 11: 147-155, 1996.

23. Hamilton HE, Gordon CPD, Nelson M, Kerbleski M. Physicians, nonphysician healthcare providers, and patients communicating in Hepatitis C:An in-office sociolinguistic study. Gastroenterology Nursing 29: 364-370, 2006.

24. Steffenino G, Viada E, Marengo B, Canale R. Effectiveness of video-based patient information before percutaneous cardiac interventions. J Cardiovasc Med (Hagerstown) 8: 348-353, 2007.

(C) 2008 The Japanese Society of Internal Medicine http://www.naika.or.jp/imindex.html 